\title{
Distributed detection in practical wireless sensor networks via a two-step consensus algorithm
}

\author{
Edmond Nurellari*, Des McLernon* and Mounir Ghogho* ${ }^{* \dagger}$ \\ * School of Electronic and Electrical Engineering, The University of Leeds, Leeds, UK \\ ${ }^{\dagger}$ International University of Rabat, Rabat, Morocco
}

\begin{abstract}
The problem of fully distributed detection of an unknown deterministic signal observed by a wireless sensor network (WSN) is addressed. We propose a two-step distributed consensus-based detection algorithm where in the first step the sensor nodes (SNs) collaborate with their neighbors through error-free, orthogonal channels (the SNs exchange quantized information matched to the channel capacity of each link). In the second step, local 1-bit decisions generated in the first step are shared among neighbors to yield a consensus. Simulations show that our proposed quantized two-step distributed detection algorithm approaches the performance of the unquantized centralized (fusion center) detector.
\end{abstract}

\section{Introduction}

Composed of low-cost tiny sensor nodes (SNs) with limited processing capabilities and on-board power, wireless sensor networks (WSNs) spatially deployed over some geographic region (see Fig. 1) are tasked to decide on a global activity from locally collected measurements. Some strategies consider the centralized solution of a fusion center (FC) where the noisy observations from distributed SNs are collected and the FC is able to compute the test statistic in a single shot $[1-5]$. Recent publications $[6,7]$ consider $\mathrm{SN}$ collaboration in the context of distributed estimation where after the exchange of information the SNs report to the FC. However, these WSNs may not be practical as they rely on the integrity of the FC.

Alternatively, fully distributed strategies [8-13] (i.e., without a FC) where the SNs exchange local information iteratively with their neighbors, are shown to be capable of reaching a global optimum decision. Reference [8] adopts the diffusion LMS approach while [10-13] adopt the distributed consensus algorithm [14]. All these works assume ideal exchange of information among the SNs, but as the SNs are battery operated (i.e., limited energy) this assumption is unrealistic. Furthermore, practical WSN scenarios suffer from channel impairments such as fading and attenuation.

So the main contribution of this work is to provide a fully distributed two-step consensus-based detection framework with quantized test statistic exchange (SNs implement a low complexity uniform quantizer and the number of quantization bits is constrained to match the channel capacity of each link). We show that this approach (a) converges to a global decision across the network, (b) approaches the optimum centralized detector performance, and (c) achieves the global decision in a finite number of iterations. The main idea of the proposed two-step detection algorithm is to arrive at an optimum global decision at each SN by taking advantage of the spatially distributed information across the network while combating flat fading.

In Section 2 we formulate the detection problem and introduce some basic definitions from graph theory. Section 3 details the general fully distributed approach analysis and Section 4 presents the proposed two-step distributed algorithm. Then, Section 5 gives simulation results and in Section 6 we draw conclusions.

\section{Problem formulation}

Consider the problem of detecting the presence of an unknown deterministic signal $s(n)$ by a WSN consisting of $M$ spatially distributed SNs. The measurement at each SN $\left(s_{i}(n)\right)$ is further corrupted by AWGN $\left(w_{i}(n) \sim \mathcal{N}\left(0, \sigma_{i}{ }^{2}\right)\right)$ and so the detection problem can be formulated as the following binary hypotheses test:

$$
\begin{aligned}
& \mathcal{H}_{0}: y_{i}(n)=w_{i}(n) \\
& \mathcal{H}_{1}: y_{i}(n)=s_{i}(n)+w_{i}(n)
\end{aligned}
$$

where $N$ samples of the observed signal are gathered and energy estimation is performed by each SN. So the $i^{t h} \mathrm{SN}$ evaluates

$$
\tilde{T}_{i}=\sum_{n=1}^{N}\left(y_{i}(n)\right)^{2}, i=1,2, \ldots, M
$$

which for large $N$, has a distribution that can be approximated by a Gaussian [15]. Furthermore, the noise samples are assumed to be identically and independently distributed (i.i.d.) across time and space. It is not difficult to show that

$$
\begin{aligned}
& \mathrm{E}\left\{\tilde{T}_{i} \mid \mathcal{H}_{0}\right\}=N \sigma_{i}^{2}, \quad \operatorname{Var}\left\{\tilde{T}_{i} \mid \mathcal{H}_{0}\right\}=2 N \sigma_{i}^{4} \\
& \mathrm{E}\left\{\tilde{T}_{i} \mid \mathcal{H}_{1}\right\}=N \sigma_{i}^{2}\left(1+\xi_{i}\right), \operatorname{Var}\left\{\tilde{T}_{i} \mid \mathcal{H}_{1}\right\}=2 N \sigma_{i}^{4}\left(1+2 \xi_{i}\right)
\end{aligned}
$$


where $\xi_{i}=\sum_{n=1}^{N} s_{i}^{2}(n) / N \sigma_{i}^{2}$. The case where spatially distributed SNs report to the FC (i.e., the centralized approach) via dedicated parallel access channels (PAC) is investigated in $[3,5]$. Here, (equal) linear soft decision combining at the $\mathrm{FC}$ gives:

$$
T_{f}=\sum_{i=1}^{M} \tilde{T}_{i}
$$

Upon receiving the local test statistics $\left(\tilde{T}_{i}, \forall i\right)$, the FC takes the final decision:

$$
\left.\begin{array}{l}
\text { if } T_{f}<\Lambda_{f}, \text { decide } \mathcal{H}_{0} \\
\text { if } T_{f} \geq \Lambda_{f}, \text { decide } \mathcal{H}_{1}
\end{array}\right\}
$$

where $\Lambda_{f}$ is the FC detection threshold. The probability of detection $\left(P_{d}\right)$ for a fixed probability of false alarm $\left(P_{f a}\right)$ is $[16]$ :

$$
P_{d}=Q\left(\frac{Q^{-1}\left(P_{f a}\right) \sqrt{\operatorname{Var}\left\{T_{f} \mid \mathcal{H}_{0}\right\}}-\mathrm{E}\left\{T_{f} \mid \mathcal{H}_{1}\right\}+\mathrm{E}\left\{T_{f} \mid \mathcal{H}_{0}\right\}}{\sqrt{\operatorname{Var}\left\{T_{f} \mid \mathcal{H}_{1}\right\}}}\right) .
$$

Now, as (1) and (2) are only the local hypotheses, our goal is to perform reliably (i.e., with detection probability (7)) the (global) detection across $M$ limited bandwidth SNs. More precisely, we will investigate the fully distributed approach (i.e., without a FC) where each SN (tasked with the detection) shares iteratively its (current) test statistic (quantized to $q_{i}$ bits) with its neighbors. The interaction among SNs is determined by the communication topology which is given by an undirected graph $\mathcal{G}=(\mathcal{V}, \mathcal{E})$, where $\mathcal{V}=\{1,2, \ldots, M\}$ represents the set of $M$ SNs and $\mathcal{E} \subseteq$ $\mathcal{V} \times \mathcal{V}$ is the set of edges $\{i, j\}$. The graph properties can be represented by an adjacency matrix $\mathbf{E} \in \mathbb{R}^{M \times M}$ whose entries are defined as

$$
e_{i j}=e_{j i}= \begin{cases}1, & \text { if } j \in \Delta_{i} \\ 0, & \text { otherwise. }\end{cases}
$$

We denote the $i^{\text {th }} \mathrm{SN}$ neighbor set as $\Delta_{i}$ and $\left|\Delta_{i}\right|$ is the cardinality/number of neighbors. The definition of the graph Laplacian matrix $\left(\mathbf{L} \in \mathbb{R}^{M \times M}\right)$ is $\mathbf{L}=\mathbf{D}-\mathbf{E}$ with $\mathbf{D}=\operatorname{diag}\left(\left|\Delta_{1}\right|, \ldots,\left|\Delta_{M}\right|\right)$.

\section{Fully distributed approach analysis}

The centralized approach (where $M$ SNs report to FC) is susceptible to FC failure. Furthermore, forwarding the data to a single processing unit would require the $\mathrm{FC}$ to process a very large amount of information, especially when the network is composed of large numbers of SNs. Hence, distributed solutions are very attractive as the computational load splits across the network. Furthermore, the global decision can be taken at each individual SN and the system is robust against SN failure. Even though there are different distributed algorithms in the literature (average consensus, diffusion and gossip-type algorithms, to name just a few), here we will stick to the average consensus algorithm [14].
3.1 Consensus algorithm with soft information exchange

The consensus algorithm allows us to initiate some global action or decision in a fault-tolerant environment on-thefly, without choosing a $\mathrm{SN}$ in advance to act as a FC (which would introduce a single point of failure). It maintains consistency in a distributed network and when some of the SNs crash or start sending corrupted data due to a hardware failure, the algorithm guarantees robustness. At iteration $k+1$, each SN $i$ updates its test statistic $\left(T_{i}[k+1]\right)$ as follows [14]:

$T_{i}[k+1]=T_{i}[k]-\epsilon \sum_{j=1}^{M} e_{i j}\left(T_{i}[k]-T_{j}[k]\right), k \geq 0, i=1, \cdots, M$

where $0<\epsilon<1 / d_{\max }$ with $d_{\max }=\max \left(\left|\Delta_{1}\right|, \ldots,\left|\Delta_{M}\right|\right)$ and $T_{i}[0]=\tilde{T}_{i}$ in (3). The time evolution in (9) can be written as

$$
\mathbf{T}[k]=\mathbf{W}^{k} \mathbf{T}[0], k \geq 1
$$

where $\mathbf{W}=\mathbf{I}-\epsilon \mathbf{L}$ and $\mathbf{T}[k]=\left[T_{1}[k], T_{2}[k], \ldots, T_{M}[k]\right]^{T}$. The decision can be taken locally at the $i^{\text {th }} \mathrm{SN}$ at the $k^{\text {th }}$ iteration as follows:

$$
\left.\begin{array}{l}
\text { if } T_{i}[k]<\Lambda_{i}[k], \text { decide } \mathcal{H}_{0} \\
\text { if } T_{i}[k] \geq \Lambda_{i}[k], \text { decide } \mathcal{H}_{1}
\end{array}\right\}
$$

where $\Lambda_{i}[k]$ is the threshold for the $i^{t h} \mathrm{SN}$ at the $k^{\text {th }}$ iteration. The expectation and the variance under both hypotheses are given as:

$$
\begin{aligned}
\mathrm{E}\left\{T_{i}[k] \mid \mathcal{H}_{p}\right\}_{p=\{0,1\}} & =\quad\left(\mathbf{W}^{k} \mathrm{E}\left\{\mathbf{T}[0] \mid \mathcal{H}_{p}\right\}\right)_{i} \\
\operatorname{Var}\left\{T_{i}[k] \mid \mathcal{H}_{p}\right\}_{p=\{0,1\}} & =\left(\operatorname{Cov}\left\{\mathbf{T}[k] \mid \mathcal{H}_{p}\right\}\right)_{i i} \\
& =\left(\mathbf{W}^{k} \operatorname{Cov}\left(\mathbf{T}[0] \mid \mathcal{H}_{\mathrm{p}}\right) \mathbf{W}^{k}\right)_{i i}
\end{aligned}
$$

where $^{1}(\mathbf{a})_{i}$ denotes the $i^{\text {th }}$ element of vector a and $(\mathbf{A})_{i j}$ denotes the $(i, j)$ element of matrix A. For a fixed probability of false alarm (i.e., $P_{f a}^{i}[k]=P_{f a}, \forall i$ and $\forall k$ ) we estimate the threshold for the $i^{t h} \mathrm{SN}$ at the $k^{\text {th }}$ iteration as

$$
\Lambda_{i}[k]=Q^{-1}\left(P_{f a}\right) \sqrt{\operatorname{Var}\left\{T_{i}[k] \mid H_{0}\right\}}+\mathrm{E}\left\{T_{i}[k] \mid H_{0}\right\}
$$

and we can write probability of detection as before [16]:

$$
\begin{aligned}
& P_{d}^{i}[k]= \\
& Q\left(\frac{Q^{-1}\left(P_{f a}\right) \sqrt{\operatorname{Var}\left\{T_{i}[k] \mid \mathcal{H}_{0}\right\}}+\mathrm{E}\left\{T_{i}[k] \mid \mathcal{H}_{0}\right\}-\mathrm{E}\left\{T_{i}[k] \mid \mathcal{H}_{1}\right\}}{\sqrt{\operatorname{Var}\left\{T_{i}[k] \mid \mathcal{H}_{1}\right\}}}\right) .
\end{aligned}
$$

Now, (15) establishes a relationship between the probability of detection $\left(P_{d}^{i}[k]\right)$ and the iteration number $k$ at

\footnotetext{
${ }^{1}$ For a random vector $\mathbf{x}, \mathrm{E}\{\mathbf{x}\}$ denotes expectation and $\operatorname{Cov}\{\mathbf{x}\}=\mathrm{E}\left[(\mathbf{x}-\mathrm{E}\{\mathbf{x}\})(\mathbf{x}-\mathrm{E}\{\mathbf{x}\})^{T}\right]$ is the covariance matrix.
} 
the $i^{\text {th }} \mathrm{SN}$. It can be shown [14] that as $k$ gets larger for a connected network ${ }^{2}$, the performance of the distributed detector (11) approaches that of the centralized detector (6) (i.e., $\lim _{k \rightarrow \infty} P_{d}^{i}[k]=P_{d}, \forall i$, see (7) and (15)).

3.2 Quantized consensus algorithm framework with limited bandwidth

Until now, we have considered distributed detection assuming ideal exchange of information between SNs. However, the communication rate in a WSN is limited due to the fact that SNs are battery operated devices. Here we propose a scheme, where SN $i$ encodes the data (using a simple uniform quantizer with $q_{i}$ bits) prior to information exchange ${ }^{3}$. We also propose to establish a link between any two SNs $i$ and $j$ based on the (known) SNR at node $j$, i.e.

$$
\left.\begin{array}{l}
\text { if } S N R_{i j}<\Upsilon, e_{i j}=e_{j i}=0 \\
\text { if } S N R_{i j} \geq \Upsilon, e_{i j}=e_{j i}=1 .
\end{array}\right\}
$$

Now $e_{i j}$ is defined in (8), $\Upsilon$ is a SNR threshold parameter (see later) and $S N R_{i j}$ is the received signal-to-noise ratio (at $\mathrm{SN} j$ ) defined as:

$$
S N R_{i j}=\frac{p_{i j}^{t} h_{i j}^{2}}{\zeta_{0} d_{i j}^{\gamma}}
$$

Here $p_{i j}^{t}$ denotes the $i^{t h}$ to $j^{t h} \mathrm{SN}$ transmit power, $h_{i j}$ is the flat-fading gain ${ }^{4}$ between the $i^{t h}$ and $j^{\text {th }} \mathrm{SN}, \zeta_{0}$ is the variance of the AWGN at each receiving SN (assumed to be the same for simplicity), $\gamma$ is the path loss coefficient and $d_{i j}$ is the physical distance between SN $i$ and $j$ (assumed to be known). We propose to quantize with $q_{i}$ bits at SN $i$ before transmitting to $\mathrm{SN} j$ and to satisfy the capacity constraint between $\mathrm{SNs} i$ and $j$ we require:

$$
q_{i} \leq \frac{1}{2} \log _{2}(1+\Upsilon) \quad \text { bits }
$$

where we let $q_{i}=q, \forall i$. Now, $\Upsilon$ establishes a relationship between the number of bits that each SN is able to transmit to its neighbors and also the topology of the network that defines the connections between the SNs (see (16)-(18)). A large $\Upsilon$ means fewer communication links (see (16)) and so slower information diffusion across the network. However, this will be counterbalanced by an

\footnotetext{
${ }^{2} \mathrm{~A}$ connected network is any network where there is a path between every pair of SNs in the network.

${ }^{3} \mathrm{We}$ assume that $\mathrm{SNs}$ transmit their observations through a parallel access channel (PAC). This assumption is reasonable for a small-scale WSNs.

${ }^{4}$ We assume that the channel coefficients are sufficiently slowly varying to be considered constant for the time interval necessary for the network to converge, within a prescribed accuracy. This assumption is reasonable as our proposed algorithm converges sufficiently fast.
}

increase in the number of bits that each SN can transmit to its neighbors (see (18)). As a consequence, the quantization noise variance (20) becomes negligible. On the other hand, a small $\Upsilon$ establishes a more connected graph and dictates a faster information diffusion across the network. However, this allows less transmission bits resulting in an increase in the quantization noise variance. It is now clear that $\Upsilon$ establishes a relationship between transmission bits and the graph connectivity. With quantization, the time evolution in (9) now becomes:

$$
\begin{aligned}
& \bar{T}_{i}[k+1]=\bar{T}_{i}[k]-\epsilon \sum_{j=1}^{M} e_{i j}\left(\bar{T}_{i}[k]-\left[\bar{T}_{j}[k]\right]_{Q}\right) \\
= & \bar{T}_{i}[k]-\epsilon \sum_{j=1}^{M} e_{i j}\left(\bar{T}_{i}[k]-\bar{T}_{j}[k]-b_{j}[k]\right), k \geq 0, i=1, \ldots, M
\end{aligned}
$$

with $\bar{T}_{i}[0]=\tilde{T}_{i}$ in $(3)$. Now $\left[\bar{T}_{j}[k]\right]_{Q}=\bar{T}_{j}[k]+b_{j}[k]$ represents quantization and $b_{j}[k]$ is the quantization noise independent of $w_{i}(n)$ in $(1), j=1,2, \cdots M, \forall i$ and $\forall n$. Assuming $\bar{T}_{j}[k] \in[0,2 U]$ and uniform quantization then:

$$
\operatorname{Var}\left\{b_{j}[k]\right\}=\sigma_{b_{j}}^{2}=\frac{U^{2}}{3 \times 2^{2 q}}
$$

with $\mathrm{E}\left\{b_{j}[k]\right\}=0$. Defining $\boldsymbol{\psi}[k]=\left[\psi_{1}[k], \psi_{2}[k], \ldots \psi_{M}[k]\right]^{T}$ with $\psi_{i}[k]=\sum_{j=1}^{M} e_{i j} b_{j}[k]$, then (19) becomes:

$$
\overline{\mathbf{T}}[k]=\mathbf{W}^{k} \overline{\mathbf{T}}[0]+\epsilon \sum_{z=1}^{k} \mathbf{W}^{z-1} \boldsymbol{\psi}[k-z], k \geq 1
$$

where $\overline{\mathbf{T}}[k]$ is defined similar to $\mathbf{T}[k]$ in (10). The decision for the $i^{t h} \mathrm{SN}$ at the $k^{t h}$ iteration is again given in (11). The expectation and the variance for the $i^{\text {th }} \mathrm{SN}$ at the $k^{\text {th }}$ iteration under both hypotheses is given as:

$$
\begin{gathered}
\mathrm{E}\left\{\bar{T}_{i}[k] \mid \mathcal{H}_{p}\right\}_{p=\{0,1\}}=\left(\mathbf{W}^{k} \mathrm{E}\left\{\overline{\mathbf{T}}[0] \mid \mathcal{H}_{p}\right\}\right)_{i} \\
\operatorname{Var}\left\{\bar{T}_{i}[k] \mid \mathcal{H}_{p}\right\}_{p=\{0,1\}}=(\underbrace{\mathbf{W}^{k} \operatorname{Cov}\left\{\overline{\mathbf{T}}[0] \mid \mathcal{H}_{\mathrm{p}}\right\}\left(\mathbf{W}^{k}\right)}_{(\mathrm{A})})_{i i} \\
+\epsilon^{2}(\underbrace{\sum_{z=1}^{k} \mathbf{W}^{z-1} \operatorname{Cov}\{\boldsymbol{\psi}[k-z]\}\left(\boldsymbol{W}^{z-1}\right)}_{(\mathrm{B})})_{i i}
\end{gathered}
$$

where $\operatorname{Cov}\{\boldsymbol{\psi}[k-z]\}=\frac{U^{2}}{3} \operatorname{diag}\left(\frac{\left|\Delta_{1}\right|}{2^{2 q}}, \frac{\left|\Delta_{2}\right|}{2^{2 q}}, \cdots, \frac{\left|\Delta_{M}\right|}{2^{2 q}}\right)$. Now, the detection performance for the $i^{\text {th }} \mathrm{SN}$ at the $k^{\text {th }}$ iteration can be evaluated using (15) (replacing $T_{i}[k]$ by $\left.\bar{T}_{i}[k]\right)$ by substituting the expressions from (22) and (23). Note that as the dynamic system (21) evolves, the term (B) in (23) accumulates. Next we show how the detection performance for the $i^{t h} \mathrm{SN}$ at the $k^{t h}$ iteration evolves by analyzing the variance term $\left(\operatorname{Var}\left\{\bar{T}_{i}[k]\right\}\right)$ in $(23)$. 
Proposition 1. From (23), the "scaled total variance" $\frac{1}{M-1} \sum_{i=1}^{M} \operatorname{Var}\left\{\bar{T}_{i}[k]\right\}$

$\leq \operatorname{Var}_{\mathrm{k}}^{\max }\left(\frac{1}{M-1}+\lambda_{2}^{2 k}(\mathbf{W})\right)+\epsilon^{2} \sigma_{\max }^{2}\left(\frac{k}{M-1}+\frac{1-\lambda_{2}^{2 k}(\mathbf{W})}{1-\lambda_{2}^{2}(\mathbf{W})}\right)$

where $\operatorname{Var}_{\mathrm{k}}^{\max }=\max \left(\operatorname{Var}\left\{\bar{T}_{1}[k]\right\}, \cdots, \operatorname{Var}\left\{\bar{T}_{M}[k]\right\}\right), \sigma_{\text {max }}^{2}=$ $\max \left(\operatorname{Var}\left\{\psi_{1}[k]\right\}, \cdots, \operatorname{Var}\left\{\psi_{M}[k]\right\}\right)$ and $\lambda_{i}(\mathbf{W}), i=1, \cdots, M$ are the eigenvalues of $\mathbf{W}$ satisfying $\lambda_{M} \leq \lambda_{M-1} \leq \cdots<\lambda_{1}=$ 1 .

Proof. The proof can be found in Appendix A.

As $k$ becomes large, it is clear that the second term of (24) grows and the performance of the distributed algorithm using quantized test statistics does not converge across the network to its counterpart of the centralized quantized detector [3] (i.e., $\lim _{k \rightarrow \infty} P_{d}^{i}[k] \neq P_{d}$ in (13) of [3], $\forall i$ ).

Now, it is also clear that the value of $k$ establish a tradeoff between the local SNs test statistic improvement and the quantization error degradation. There is a finite $k$ to stop the SNs collaboration (see later), wheres after that value the quantization error overcomes the improvement gain from this collaboration. So using this framework (i.e., the consensus algorithm with quantization matched to the channel capacity) we will now propose a twostep approach (still using quantized test statistics shared among SNs) that will now approach the performance of the optimum centralized detector (6) (i.e., when using a FC) and what is more important, it will converge across the whole network.

\section{Proposed two-step fully distributed detection}

(i) FIRST STEP: We will run the quantized consensus algorithm in (21) to improve the local version of the test statistic at each SN. But now we will terminate the algorithm at $k=K_{1}$ (where the optimum ${ }^{5}$ value of $K_{1}$ is found later from simulation results). We now have $\left\{\bar{T}\left[K_{1}\right]\right\}_{i=1}^{M}$ from $(21)$ and we will use this to generate a binary indicator random variable $I_{i}[0]$ as follows

$$
\left.\begin{array}{l}
\text { if } \bar{T}_{i}\left[K_{1}\right]<\Lambda_{1}, I_{i}[0]=0 \\
\text { if } \bar{T}_{i}\left[K_{1}\right] \geq \Lambda_{1}, I_{i}[0]=1
\end{array}\right\}
$$

where $\Lambda_{1}$ is a local (first step) detection threshold that is the same for all $M$ SNs. We will adopt the following global decision rule

$$
\left.\begin{array}{l}
\text { if } \bar{T}_{f}\left[K_{1}\right] \neq M \text {, decide } \mathcal{H}_{0} \\
\text { if } \bar{T}_{f}\left[K_{1}\right]=M \text {, decide } \mathcal{H}_{1}
\end{array}\right\}
$$

\footnotetext{
${ }^{5}$ Based on the simulation results, we find a finite $K_{1}$ (to stop the collaboration) that is "optimum" in the sense that $P_{d}$ in (7) (i.e., effective upper bound) is sufficiently well approximated (i.e., using $\overline{\mathbf{T}}\left[K_{1}\right]$ ) and after that (i.e., for larger $K_{1}$ ) this approximation degrades.
}

where $\bar{T}_{f}\left[K_{1}\right]=\sum_{i=1}^{M} I_{i}[0]$. But the problem is now how to evaluate $\bar{T}_{f}\left[K_{1}\right]$ in a distributed manner across the SNs. This will be explained in step 2 .

(ii) SECOND STEP: Here we will use [17] to show how to effectively evaluate (26) by first sharing $\left\{I_{i}[0]\right\}_{i=1}^{M}$ and then iteratively updating across the SNs as follows:

$$
\begin{array}{r}
I_{i}[k+1]=I_{i}[k] \bigwedge\left(\bigwedge_{j \in \Delta_{i}} I_{j}[k]\right), k=0,1,2, \cdots, K_{2}-1, \\
i=1,2, \cdots, M \quad(27)
\end{array}
$$

where $K_{2}$ is the diameter of network ${ }^{6}$, "^" denotes the logical "and" operation and $\Delta_{i}$ is defined for (8). Note that no quantization is needed and all $I_{i}\left[K_{2}\right]$ converge to either 1 or 0 . So now we can easily show:

$$
\left.\begin{array}{l}
\text { If } I_{i}\left[K_{2}\right]=0, \forall i \Rightarrow \bar{T}_{f}\left[K_{1}\right] \neq M \text {, decide } \mathcal{H}_{0} \\
\text { If } I_{i}\left[K_{2}\right]=1, \forall i \Rightarrow \bar{T}_{f}\left[K_{1}\right]=M \text {, decide } \mathcal{H}_{1} .
\end{array}\right\}
$$

and so $I_{i}\left[K_{2}\right]$ (at any SN) can be used to implement the decision rule (26). Overall, the proposed two-step fully distributed algorithm requires a finite $\left(K_{1}+K_{2}\right)$ iterations in total.

\section{Simulations results}

Here we will analyze the performance of our proposed two-step distributed detection algorithm. First we have a WSN with $M$ SNs with arbitrary SN geometry, where the distances $d_{i j}$ in (17) between SNs $i$ and $j$ are assumed to be known. The other parameters in (17) are $p_{i j}^{t}=300$, $\gamma=2, \zeta_{0}=0.1$ and $h_{i j}^{2}$ is an exponential random variable (r.v.). Using the r.v. $S N R_{i j}$ of (17) in (16), we then construct two example topologies for different values of $\Upsilon$ (see Fig. 1). These topologies will be used later for Fig. 4 and Fig. 5. We now generate the test statistics $\bar{T}_{i}\left[K_{1}\right]$ in (25), via (21) for $k=K_{1}$. As previously explained, any $I_{i}\left[K_{2}\right]$ in $(28)$ can be used to decide either $\mathcal{H}_{0}$ or $\mathcal{H}_{1}$, and this will define the new global detection and false alarm probabilities (i.e., $P_{d}^{g}$ and $P_{f a}^{g}$ respectively). Here we use $10^{5}$ Monte-Carlo simulations. Finally, $\xi_{a}=$ $10 \log _{10}\left(\frac{1}{M} \sum_{i=1}^{M} \xi_{i}\right)=-9.5 \mathrm{~dB}$ (with all $\sigma_{i}^{2}$ terms different for each $\xi_{i}$ ) in Figs 2-5.

Fig. 2 shows the global detection performance $\left(P_{d}^{g}\right)$ for a fixed probability of false alarm $\left(P_{f a}^{g}\right)$ parametrized against $\Upsilon$. It is clear that as the number $(M)$ of SNs increases $P_{d}^{g}$ improves. Furthermore, it is interesting to observe that there exists an optimum $\Upsilon$ such that $P_{d}^{g}$ can be maximized

${ }^{6}$ The geodesic distance between two nodes in a (connected) graph is the number of the edges (i.e., links) in the shortest path connecting these two nodes. The diameter of a graph is the maximum geodesic distance taken over all possible pairs of nodes in the graph. 

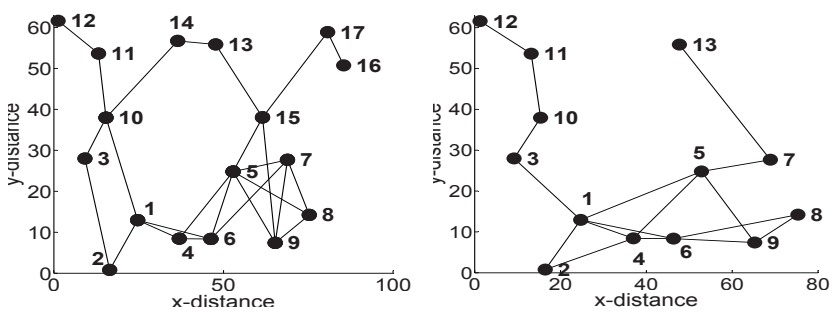

Figure 1. Two different communication topologies (generated via ((16) and (17)), with the quantization bits following (18): (left) $M=17, \Upsilon=20, q=2$ bits; (right) $M=13, \Upsilon=72, q=3$ bits.

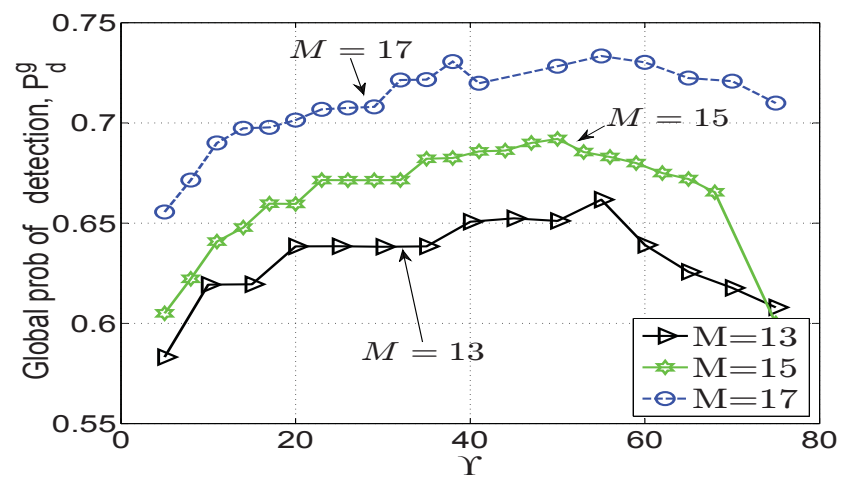

Figure 2. Global probability of detection $\left(P_{d}^{g}\right)$ (using two step approach) versus $\Upsilon$ in (16), with $P_{f a}^{g}=0.2, U=2, N=20$ $K_{1}=10$.

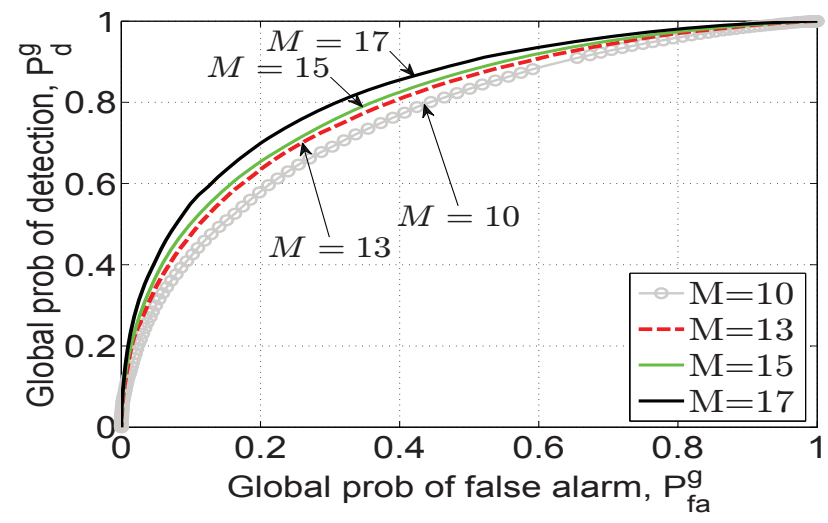

Figure 3. Receiver operating characteristic for the proposed two step distributed scheme with $\Upsilon=20$ in (16), $U=2, N=20$ and $K_{1}=10$.

for any $M$. In Fig. 3, we examine the receiver operating characteristic (ROC) parametrized against $M$ for the proposed two-step algorithm, illustrating how $P_{d}^{g}$ improves as $M$ increases. The ROC performance ${ }^{7}$ among different schemes is illustrated in Fig. 4 and Fig. 5, where Fig. 4 shows the advantage of our proposed distributed twostep scheme over only the first step part (at SN 6). Also, if $\Upsilon$ is carefully chosen the two-step proposed scheme performance approaches that of the centralized detector

${ }^{7}$ SN 6 (Fig. 4) and SN 3 (Fig. 6) were chosen for comparison purposes as they possess the best performances among $M$ SNs for each case.

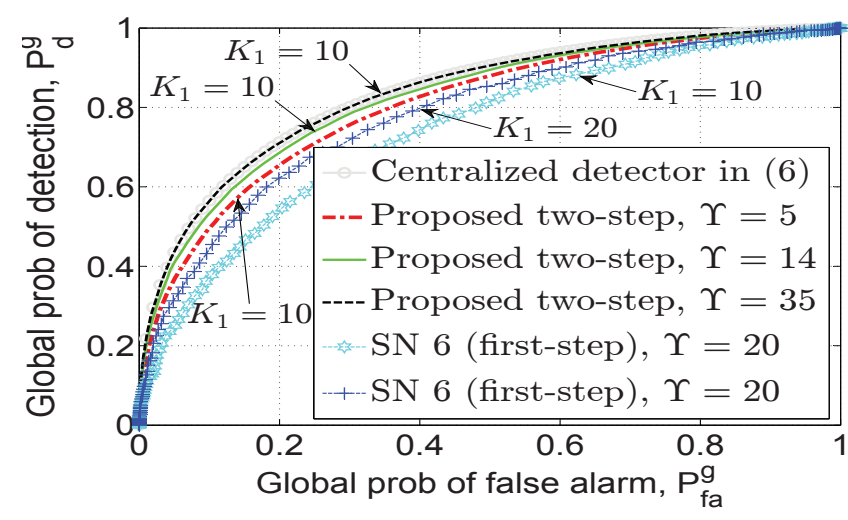

Figure 4. Receiver operating characteristic with $U=2, N=20$, $M=17$ and topology given in left of Fig. 1 .

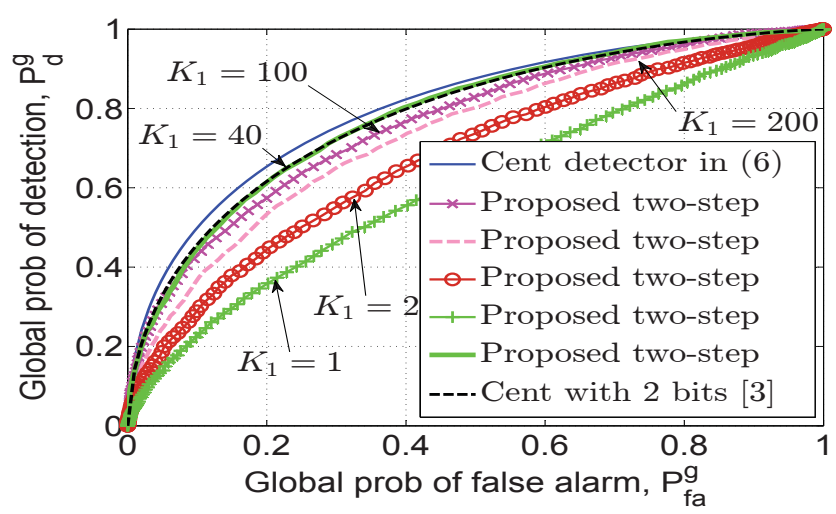

Figure 5. Receiver operating characteristic with $U=2, N=20$, $M=13$ and topology given in right of Fig. 1 .

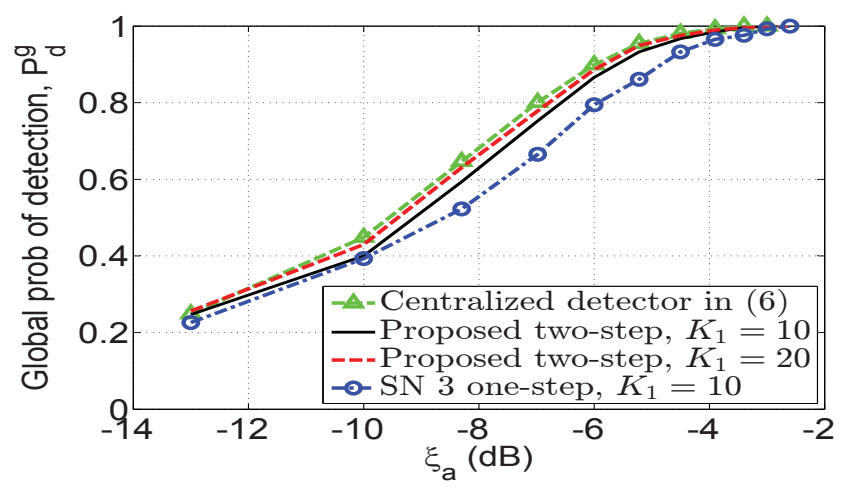

Figure 6. Probability of detection $\left(P_{d}^{g}\right)$ versus the signal to noise ratio $\left(\xi_{a}\right)$ for $M=13, \Upsilon=72, U=2, N=20, P_{f a}^{g}=0.1$ and $\xi_{i}=\xi . \forall i$ in (4). The topology used is given in right of Fig. 1.

(i.e., with FC and no quantization) in (6). Fig. 5 shows the behavior of the proposed distributed two-step algorithm compared to the quantized ( 2 bits) equal combining scheme in [3]. As expected, there is an optimum $K_{1}$ that maximizes $P_{d}^{g}$ and after that $P_{d}^{g}$ decreases. Finally, Fig. 6 shows the $P_{d}^{g}$ performance characterization against the average $\operatorname{SNR}\left(\xi_{a}\right)$ for 4 different schemes showing the performance improvement of our proposed scheme. 


\section{Conclusion}

In this paper, we propose a fully distributed two-step consensus-based detection algorithm via $\mathrm{SNs}$ sharing with their neighbors a quantized version of the received energy test statistic. We relate the communication topology with the number of bits to be shared among SNs and through numerical results we show that there is an optimum topology (for a fixed first step number of iterations $\left.\left(K_{1}\right)\right)$ such that $P_{d}^{g}$ (the global probability of detection) is maximized. In addition, we show that there is an optimum $K_{1}$ to terminate the first step SN collaboration (for any arbitrary topology) and after that the $P_{d}^{g}$ performance declines. When $K_{1}$ and $\Upsilon$ (SNR threshold in (16)) are appropriately chosen, the detection performance of the proposed quantized two-step detector approaches the centralized unquantized equal combining scheme performance of (6). Overall, our algorithm requires a finite number of iterations, $\left(K_{1}+K_{2}\right)$. While the assumed proposal was based on the (non-optimal) equal combining strategy of (5), future work will develop a similar twostep approach based on optimal linear combining of the test statistics.

\section{A Appendix. Proof of Proposition 1}

Consider (24):

$$
\begin{gathered}
\frac{1}{M-1} \sum_{i=1}^{M} \operatorname{Var}\left\{\bar{T}_{i}[k]\right\}=\frac{1}{M-1} \operatorname{tr}\left(\operatorname{Cov}\left\{\overline{\mathbf{T}}[k] \mid H_{p}\right\}_{\{p=0,1\}}\right) \\
=\frac{1}{M-1} \operatorname{tr}\left(\left(\mathbf{W}^{k} \operatorname{Cov}\left(\overline{\mathbf{T}}[0] \mid \mathrm{H}_{\mathrm{p}}\right) \mathbf{W}^{k}\right)\right. \\
\quad+\frac{\epsilon^{2}}{M-1} \operatorname{tr}\left(\sum_{z=1}^{k} \mathbf{W}^{z-1} \operatorname{Var}\{\boldsymbol{\psi}[k-z]\} \mathbf{W}^{z-1}\right) \\
\leq \frac{1}{M-1}\left(\operatorname{Var}_{\mathrm{k}}^{\max ^{2}} \operatorname{tr}\left(\mathbf{W}^{2 k}\right)+\epsilon^{2} \sigma_{\max }^{2} \operatorname{tr}\left(\sum_{z=1}^{k} \mathbf{W}^{2 z-2}\right)\right)
\end{gathered}
$$

where $\operatorname{tr}($.$) denotes the trace operator, \operatorname{Var}_{\mathrm{k}}^{\max }=$ $\max \left(\operatorname{Var}\left\{\bar{T}_{1}[k]\right\}, \operatorname{Var}\left\{\bar{T}_{2}[k]\right\}, \cdots, \operatorname{Var}\left\{\bar{T}_{M}[k]\right\}\right) \quad$ and $\sigma_{\text {max }}^{2}=\max \left(\operatorname{Var}\left\{\psi_{1}[k]\right\}, \cdots, \operatorname{Var}\left\{\psi_{M}[k]\right\}\right)$. Now we can show that

$$
\begin{gathered}
\frac{1}{M-1}\left(\operatorname{Var}_{\mathrm{k}}^{\max } \operatorname{tr}\left(\mathbf{W}^{2 k}\right)+\epsilon^{2} \sigma_{\max }^{2} \operatorname{tr}\left(\sum_{z=1}^{k} \mathbf{W}^{2 z-2}\right)\right) \\
\leq \operatorname{Var}_{\mathrm{k}}^{\max }\left(\frac{1}{M-1}+\lambda_{2}^{2 k}(\mathbf{W})\right) \\
+\epsilon^{2} \sigma_{\max }^{2}\left(\frac{k}{M-1}+\frac{1-\lambda_{2}^{2 k}(\mathbf{W})}{1-\lambda_{2}^{2}(\mathbf{W})}\right)
\end{gathered}
$$

where $\lambda_{i}(\mathbf{W})$, for $i=1,2, \cdots, M$ are the eigenvalues of W satisfying $\lambda_{M} \leq \lambda_{M-1} \leq \cdots<\lambda_{1}=1$ and we have

$$
\begin{aligned}
\text { used } \operatorname{tr}(\mathbf{W}) & =\sum_{i=1}^{M} \lambda_{i}(\mathbf{W}) \text { and } \\
\sum_{z=1}^{k} \lambda_{i}^{z}(\mathbf{W}) & =\left\{\begin{array}{cc}
\frac{\lambda_{i}(\mathbf{W})-\lambda_{i}^{k+1}(\mathbf{W})}{1-\lambda_{i}(\mathbf{W})}, & \text { for } i=2,3, \cdots, M \\
k, & \text { for } i=1 .
\end{array}\right.
\end{aligned}
$$

\section{References}

[1] J. N. Tsitsiklis, "Decentralized detection," In Advances in Statistical Signal Processing: vol 2 - Signal Detection," H. V. Poor, and John B. Thomas, eds., JAI Press, Greenwich, CT, pp. 297-344, Nov. 1993.

[2] R. Niu, B. Chen and P. K. Varshney, "Fusion of decisions transmitted over Rayleigh fading channels in wireless sensor networks," IEEE Transactions on Signal Processing, vol. 54, pp.1018-1027, 2006.

[3] E. Nurellari, D. McLernon, M. Ghogho and S. Aldalahmeh, "Optimal quantization and power allocation for energy-based distributed sensor detection," Proc. EUSIPCO, Lisbon, Portugal, 1-5 Sept. 2014.

[4] S. Barbarossa, S. Sardellitti, and P. Di Lorenzo, "Distributed Detection and Estimation in Wireless Sensor Networks," In Rama Chellappa and Sergios Theodoridis eds., Academic Press Library in S. Proc, vol. 2, Communications and Radar Signal Processing, pp. 329-408, 2014.

[5] E. Nurellari, S. Aldalahmeh, M. Ghogho and D. McLernon, "Quantized Fusion Rules for Energy-Based Distributed Detection in Wireless Sensor Networks," Proc. SSPD, Edinburgh, Scotland, 8-9 Sept. 2014.

[6] S. Kar and P. K. Varshney, "A decentralized framework for linear coherent estimation with spatial collaboration," Proc. ICASSP, Florence, Italy, 4-9 May. 2014.

[7] M. Fanaei, M. C. Valenti, A. Jamalipour and N. A. Schmid, "Optimal power allocation for distributed blue estimation with linear spatial collaboration," Proc. ICASSP, Florence, Italy, 4-9 May. 2014.

[8] F. Cattivelli and A. H. Sayed, "Diffusion LMS strategies for distributed estimation," IEEE Trans. Signal Processing, vol. 58, no. 3, pp. 1035-1048, Mar. 2010.

[9] F. Iutzeler and P. Ciblat, "Fully distributed signal detection: application to cognitive radio," Proc. EUSIPCO, Marrakech, Morocco, 9-13 Sept. 2013.

[10] I. D. Schizas, G. Mateos, and G. B. Giannakis, "Distributed LMS for consensus-based in-network adaptive processing," IEEE Trans. Signal Processing, vol. 8, no. 6, pp. 2365-2381, Jun. 2009.

[11] A. Bertrand and M. Moonen, "Distributed computation of the Fiedler vector with application to topology inference in ad hoc networks," Signal Processing, vol. 93, no.5, pp. 1106-1117, May 2013.

[12] S. Kar and J. M. F. Moura, "Topology for Distributed Inference on Graphs," IEEE Trans. Signal Process., vol. 56, pp. 2609-2613, 2008

[13] W. Zhang, Z. Wang, Y. Guo, H. Liu, Y. Chen and J. Mitola, "Distributed cooperative spectrum sensing based on weighted average consensus," Proc. GLOBECOM, Houston, Texas, USA, pp. 5-9, Dec. 2011.

[14] R. O. Saber, J. A. Fax, R. M. Murray, "Consensus and cooperation in networked multi-agent systems," Proc. of the IEEE, vol. 95, no.1, pp. 215-233, 2007.

[15] H. Urkowitz, "Energy detection of unknown deterministic signals," Proc. IEEE , vol. 55, pp. 523-531, Apr. 1967.

[16] S. M. Kay, Fundamentals of Statistical Signal Processing: Detection Theory, Englewood Cliffs, NJ: PrenticeHall PTR, 1993.

[17] S. Zheng, X. Yang and C. Lou, "Distributed consensus algorithms for decision fusion based cooperative spectrum sensing in cognitive radio," Communications and Information Technologies (ISCIT), Hangzhou, China, 12-14 Oct. 2011 